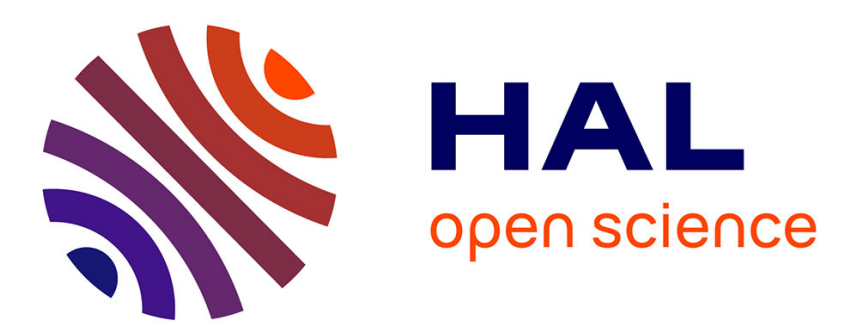

\title{
Magnetic drop-sheath wetting transition of a ferrofluid on a wire
}

\author{
J.-C. Bacri, C. Frenois, R. Perzynski, D. Salin
}

\section{To cite this version:}

J.-C. Bacri, C. Frenois, R. Perzynski, D. Salin. Magnetic drop-sheath wetting transition of a ferrofluid on a wire. Revue de Physique Appliquée, 1988, 23 (6), pp.1017-1022. 10.1051/rphysap:019880023060101700 . jpa-00245902

\section{HAL Id: jpa-00245902 https://hal.science/jpa-00245902}

Submitted on 1 Jan 1988

HAL is a multi-disciplinary open access archive for the deposit and dissemination of scientific research documents, whether they are published or not. The documents may come from teaching and research institutions in France or abroad, or from public or private research centers.
L'archive ouverte pluridisciplinaire HAL, est destinée au dépôt et à la diffusion de documents scientifiques de niveau recherche, publiés ou non, émanant des établissements d'enseignement et de recherche français ou étrangers, des laboratoires publics ou privés. 


\title{
Magnetic drop-sheath wetting transition of a ferrofluid on a wire
}

\author{
J.-C. Bacri, C. Frenois, R. Perzynski and D. Salin \\ Laboratoire d'Ultrasons (*), Université Pierre et Marie Curie, Tour 13, 4 place Jussieu, 75252 Paris Cedex 05, \\ France
}

(Reçu le 22 octobre 1987, révisé le 15 janvier 1988, accepté le 28 janvier 1988)

\begin{abstract}
Résumé. - Nous étudions l'étalement d'une goutte de ferrofluide sur un fil. L'étalement du liquide magnétique non mouillant est piloté par le champ magnétique créé par application d'un courant électrique dans le fil conducteur. Pour un courant seuil, nous prédisons un étalement de la goutte sous une forme de manchon. Cette transition de mouillage magnétique est analogue à la transition de mouillage sur une fibre prédite pour un liquide totalement mouillant en présence de forces de Van der Waals. Nous montrons des expériences préliminaires en accord avec nos prédictions théoriques.
\end{abstract}

\begin{abstract}
We study the spreading of a non wetting ferrofluid drop along a wire. The external control parameter of the magnetic fluid spreading is the magnetic field generated by the current in the conducting wire. For a current threshold we predict a spreading of the drop into a sheath coverage. This magnetic wetting transition is analogous to the wetting transition on a fiber predicted for totally wetting fluid in presence of Van der Waals forces.
\end{abstract}

\section{Introduction.}

After pioneering works on wetting of solids by fluids two centuries ago [1], it is only recently that many efforts have been paid to determine completely the spreading of a drop on a flat surface [2, 3, 4]. The socalled spreading parameter $S$ :

$$
S=\gamma_{\mathrm{SO}}{ }^{-}-\gamma_{\mathrm{SL}}-\gamma
$$

where $\gamma_{\mathrm{SO}}, \gamma_{\mathrm{SL}}$ and $\gamma$ are the interfacial tensions between solid/liquid and liquid/fluid, controls the wetting of the surface : $S<0$ corresponds to partial spreading; the drop forms a nearly spherical cap with a contact angle $\theta\left(\gamma \cos \theta=\gamma_{\mathrm{SO}}-\gamma_{\mathrm{SL}}\right)$. $S>0$ corresponds to complete wetting; the drop spreads over the surface in order to achieve a pancake shape analysed by de Gennes [2] and Joanny [3]. The thickness $e$ of this pancake results from a balance between spreading power $S$ and disjoining pressure due to long range Van der Waals forces. The typical size of this film $(e=$ $b \sqrt{3 \gamma / 2 S}$ ), is generally of the order of molecular size $b$ except in the close vicinity of a wetting transition $(S \rightarrow 0)$.

(*) Associated with the Centre National de la Recherche Scientifique.
Wetting of fibers is of particular interest for applications in textil and cosmetic industries. This problem has been recently reconsidered in details by Brochard [5] : due to the axial geometry of the fiber the liquid does not spread out at $S=0$ but at a sensitively higher threshold $S_{\mathrm{c}}$. At the threshold the drop spreads into a sheath like coverage (manchon) of typical thickness of a few nanometers. Some experimental evidences of this wetting transition has been reported [6,7], but because of the molecular thickness of the involved film the experiment is of hard observation and is very sensitive to surface roughness.

In a previous paper [8], we have studied the ascension of a ferrofluid along a vertical wire. In such an experiment the control parameter is the magnetic field generated by the current in the conducting wire [9]. A magnetic wetting transition is predicted and clearly experimentally observed [8] : at the current threshold the ferrofluid raises up along the wire with a sheath-like coverage. The sheath thickness results from a balance between the negative $S$ parameter and the magnetic disjoining pressure which monitors the spreading of the ferrofluid : the sheath thickness is of the order of the wire diameter $(\sim 100 \mu \mathrm{m})$. The phenomenon is then macroscopic $[10,11]$. 
In the present paper, we discuss in details the existence of a magnetic drop/sheath transition and the continuous or discontinuous character of this transition. We then report on first experimental observation of such a transition.

\section{Theory.}

The spreading of a drop on a thin cylinder, at $S>0$, was studied by Brochard [5] leading to the prediction of a wetting transition from a droplet to a sheath coverage of the cylinder. The sheath thickness is of the order of nanometers. Our theoretical approach follows the main trends of this article with magnetic forces in place of Van der Waals forces. This allows us to predict a magnetic wetting transition for ferrofluids with $S<0$.

The conducting wire is a cylinder of radius $a$, parallel to the $z$-direction and we are interested in the profile of the magnetic fluid drop $r(z)$ ( $r$ : radial distance from the cylinder axis) as a current of intensity $I$ is travelling through the wire ; the corresponding magnetic field at a distance $r$ is $\mu_{0} I / 2 \pi a$ where $\mu_{0}=4 \pi \times 10^{-7}$ is the vacuum permeability. The ferrofluid is characterized by a magnetic susceptibility $\chi$ at low field. The ferrofluid droplet is immersed in a non magnetic surrounding liquid of same density than the ferrofluid. For a monotonous profile $0<\theta \leqslant \pi / 2$ (cf. appendix I where the more general situation $0<\theta<\pi$ is discussed), the free energy of the system is :

$$
\begin{aligned}
F=2 \pi \int \mathrm{d} z & \left(\gamma r \sqrt{1+r^{\prime 2}}-\right. \\
& \left.-\left(\gamma_{\mathrm{SO}}-\gamma_{\mathrm{SL}}\right) a-\frac{\mu_{0} \chi I^{2}}{8 \pi^{2}} \log \frac{r}{a}\right)
\end{aligned}
$$

where $r^{\prime}=\mathrm{d} r / \mathrm{d} z$, the different interfacial tensions have been defined in the introduction ( $\mathrm{S}$ : solid, $\mathrm{L}$ : magnetic liquid, 0 : surrounding fluid). The first two terms of (2) account for capillarity, the third one for magnetism. The free energy $F$ must be minimal for all variations of the profile which conserve the total volume of the drop : $\Omega=\pi \int\left(r^{2}-a^{2}\right) \mathrm{d} z$. Using a Lagrange multiplier $p_{0}$, which may be interpreted as the pressure difference between the magnetic fluid and the surrounding one, we minimize $\mathcal{L}=F$ $p_{0} \Omega$ at constant volume $\Omega$. One obtains :

$$
\frac{1}{r\left(1+r^{\prime 2}\right)^{1 / 2}}-\frac{r^{\prime \prime}}{\left(1+r^{\prime 2}\right)^{3 / 2}}-\frac{H}{r^{2}}-\frac{p_{0}}{\gamma}=0
$$

where $H=\mu_{0} \chi I^{2} / 8 \pi^{2} \gamma$ is a characteristic magnetic length. It is straightforward to verify that :

$$
\frac{r}{\sqrt{1+r^{\prime 2}}}-a \cos \theta-H \log \frac{r}{a}-\frac{p_{0}}{2 \gamma}\left(r^{2}-a^{2}\right)=0
$$

is a first integral of (3) with the boundary condition at the wire surface $r^{\prime}=-\operatorname{tg} \theta$ for $r=a$. As Van der Waals forces are neglected our discussion is limited to $\theta \neq 0(S<0)$. In (4) $p_{0}$ value is determined from droplet volume conservation after computation of the equilibrium profiles. We first briefly recall the main features of the simple case $p_{0}=0$ which corresponds to an infinitely large drop and is equivalent to the ascension along a wire.

\subsection{ASCENSION OF A FERROFLUID ALONG A VERTI-} CAL WIRE $\left(p_{0}=0\right)$.

2.1.1 Sheath coverage. - Let us assume that one can reach a regime where the magnetic liquid spreads along the wire leading to a sheath of uniform radius $r\left(r^{\prime 2} \ll 1, r^{\prime \prime} \ll r^{-1}\right)$. We get from (3)

$$
r=H
$$

i.e. the radius of the sheath is the magnetic length. Contrary to the Van der Waals sheath (5) which is of the order of a few nanometers, our magnetic sheath is of the order of the wire radius $a$.

2.1.2 Magnetic wetting transition. - An ultimate integration of (4), with $p_{0}=0$, gives the profile $z(r)$ of the interface :

$$
\begin{aligned}
|z|=\int_{a}^{r} \mathrm{~d} r & \left(a \cos \theta+H \log \frac{r}{a}\right) / \\
& /\left(r^{2}-\left(a \cos \theta+H \log \frac{r}{a}\right)^{2}\right)^{1 / 2}
\end{aligned}
$$

If the wire current is increased from zero to a low value ( $H<a$ for instance), the magnetic liquid raises up along the wire. If $I$ is further increased, computation of (6) is only possible up to a critical magnetic length $H_{\mathrm{S}}$ where $(6)$ diverges $\left(r^{\prime 2} \leqslant 0\right)$. At this magnetic threshold the fluid raises up without bond along the wire with a sheath like coverage of thickness $r_{\mathrm{S}}$ given by :

$$
r_{\mathrm{S}}-H_{\mathrm{S}} \log \frac{r_{\mathrm{S}}}{a}-a \cos \theta=0
$$

Using the sheath condition (5) $\left(H_{\mathrm{S}}=r_{\mathrm{S}}\right)$ one obtains the relationship between the contact angle $\theta$ and the critical magnetic length $H_{\mathrm{S}} . r_{\mathrm{S}}$ is the sheath radius corresponding to complete spreading of the magnetic fluid all along the cylindrical wire : this magnetic wetting is analogous to the wetting transition predicted by Brochard in case of Van der Waals forces with $S>0$. We have already reported [8] on the experimental evidence of this transition for a ferro, fluid.

\subsection{MAGNETIC SPREADING OF A FERROFLUID DROP ALONG A WIRE.}

2.2.1 Sheath coverage. - Let us again assume that one can reach a regime where the magnetic drop 
spreads along the wire with a uniform radius $r$ $\left(r^{\prime 2} \ll 1, r^{\prime \prime} \ll r^{-1}\right.$, except in the tip). We get from (3) :

$$
\frac{p_{0}}{\gamma}=\frac{1}{r}\left(1-\frac{H}{r}\right) .
$$

2.2.2 Magnetic sheath; droplet wetting transition. An ultimate integration of (4), whatever $p_{0}$, gives the profile $z(r)$ of the interface :

$$
|z|=\int_{a}^{r}-\frac{\mathrm{d} r}{r^{\prime}}
$$

With $\quad r^{\prime}=-\left[r^{2}-\left(a \cos \theta+H \log \frac{r}{a}+\frac{p_{0}}{2 \gamma}\left(r^{2}-a^{2}\right)\right)^{2}\right]^{1 / 2} /\left(a \cos \theta+H \log \frac{r}{a}+\frac{p_{0}}{2 \gamma}\left(r^{2}-a^{2}\right)\right)$.

The pressure $p_{0}$ of the drop has to be determined from conservation of its volume :

$$
\Omega=2 \pi \int_{a}^{L} \mathrm{~d} r\left(r^{2}-a^{2}\right) / r^{\prime}
$$

$L$ is the apex of the drop (maximum $r$ value along the profile, $r=L, z=0$ ); the singularity $r^{\prime}=0$ for $r=L$ is easily removed in the integration of (9) or (10). Because of the required adjustment of $p_{0}$ through (10), the discussion is somewhat difficult : as the magnetic length $H$ is increased, there is a decrease of both $L$ and $p_{0}$ as the drop flattens along the wire. In order to make the discussion of the possibility of a drop/sheath transition more illuminating, we anticipate on the fact that a sheath corresponds to $r^{\prime}=0$ and leads to a singularity in expressions (9) and (10). The relationship, for $r^{\prime}=0$, between the magnetic length $H$ and the radius $r$ is :

$$
H=\left(r-a \cos \theta-\frac{p_{0}}{2 \gamma}\left(r^{2}-a^{2}\right)\right) / \log \frac{r}{a} .
$$

In figures 1 and 3 , the right hand side (RHS) of (11) is sketched versus $r$ for $\theta=80^{\circ}$, corresponding to our experimental conditions, at various normalized pressures $P=p_{0} a / 2 \gamma$. The choice of $\theta(0<\theta<$ $\pi$, cf. appendix I) is not very crucial, it only modifies the numerical values but not the main features of figures 1 and 3 . In such a diagram, solutions of (11) are the intersections of horizontal straight line of ordinate $H$ with the isobaric curve $P$. Depending on the monotonous character of the RHS of (11) versus $r$, the related isobars can be separated in two sets : for $P>P_{\mathrm{c}}$ isobars are decreasing functions of $r$ whereas for $P<P_{\mathrm{c}}$ the isobars exhibit a minimum ; $P_{\mathrm{c}}$ is the $\theta$ dependent normalized pressure for which the minimum is also an inflection point $\left(r=r_{\mathrm{c}}\right.$, $\left.P_{\mathrm{c}}=a / 4 r_{\mathrm{c}}\right)$.

2.2.2a Large drops (Figs. 1 and 2). - In this regime $\left(P<P_{\mathrm{c}}\right)$ every curve exhibits a minimum. Simple calculations show that the $H$ values of these minima fulfil the sheath condition (8); whence we conclude that these minima are a sequence of sheath coverage (dashed line in Figs. 1 and 3). For low $H$ values there is only one intersection between horizontal and the drop isobar $P$ : it is the trivial solution $r=L$, the drop apex. For example, the drop of figure 2 has an REVUE DE PHYSIQUE APPLIQUÉE. - T. 23, N` 6, JUIN 1988

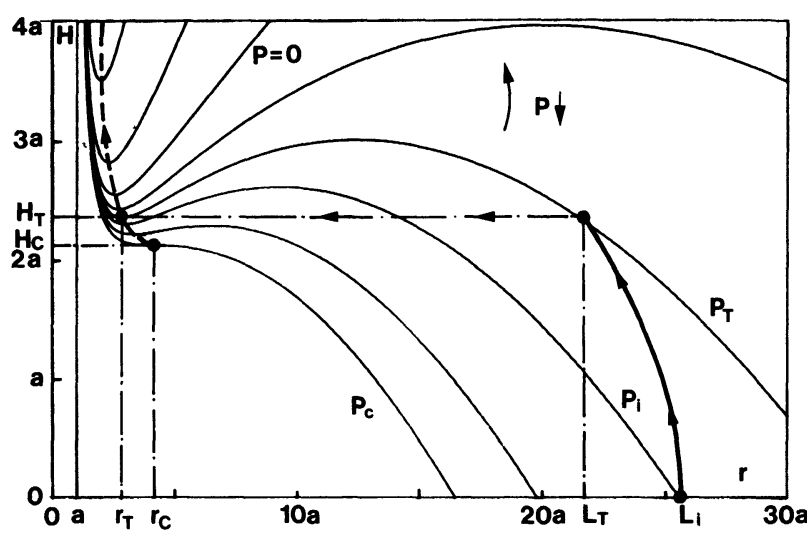

Fig. 1. - Spreading of a large magnetic drop along a conducting wire as the magnetic length $H$ is increased (cf. Fig. 2) : thick full-line : evolution of the apex $L$ computed from relations (9) and (10) ; full lines : isobars from RHS of relation (11) at constant normalized pressure $P=p_{0} a / 2 \gamma$ with $\theta=80^{\circ}$; dashed curve : sheath coverage condition (relation (8)). Subscript $i$ is related to initial drop conditions; subscript $T$ is related to threshold conditions. At the threshold, there is a jump in the drop profile leading to a sheath coverage of thickness $r_{\mathrm{T}}$ : the magnetic wetting transition of a large drop is discontinuous ; subscript $c$ is related to the end point of the sheath coverage (cf. critical drop section). Numerical values are given in the text and in caption of figure 2.

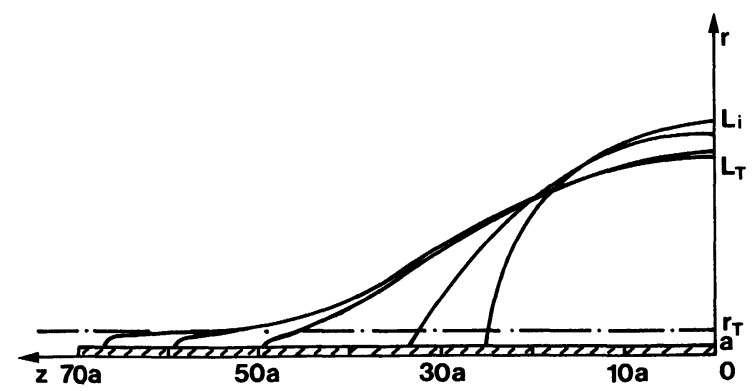

Fig. 2. - Computed profiles of a magnetic drop spreading along a conducting wire: large drop of initial apex $L_{\mathrm{i}}=26 a$ and initial normalized pressure $P_{\mathrm{i}}=0.65 P_{\mathrm{c}}$ $\left(P_{\mathrm{c}}=0.06\right)$. The contact angle $\theta$ is $80^{\circ}$. Different curves correspond to various current intensities and thus to different magnetic lengths $H: 1-\left(H / H_{\mathrm{T}}\right)=1,5 \times 10^{-1}$, $5 \times 10^{-2}, 5 \times 10^{-3}, 5 \times 10^{-4} \cdot H_{\mathrm{T}}=2.34 a$ is the magnetic length threshold of the magnetic wetting transition leading to a sheath coverage of thickness $r_{\mathrm{T}}=2.8 a . L_{\mathrm{T}}=22 a$ is the drop apex at the threshold and $P_{\mathrm{T}}=0.51 P_{\mathrm{c}}$ its normalized pressure. 
initial pressure $P_{\mathrm{i}}=0.65 P_{\mathrm{c}}$ and its zero field initial apex $L_{\mathrm{i}}=26 a$ corresponds to the intersection of the isobar $P_{\mathrm{i}}$ with the $x$ axis in figure 1 . It is then straightforward to describe the behaviour of such a large drop as $H$ is increased from zero : the thick full-line in figure 1 is deduced from the computed profiles (Fig. 2) : at low $H$ values (i.e. low current, there is only the apex $L$ solution. The larger $H$, the smaller $L$, the smaller $P$; in figure 1 the full-line displays this small $P$ decrease from $P_{\mathrm{i}}$ which is followed from volume conservation [10] as the profile is computed. This monotonous flattening of the drop on the wire occurs until a critical magnetic length $H_{\mathrm{T}}\left(H_{\mathrm{T}}=2.34 a\right.$ in our example $)$ at which the horizontal line $H_{\mathrm{T}}$ intersects the drop isobar $\left(P_{\mathrm{T}}=\right.$ $\left.0.51 P_{\mathrm{c}}\right)$ at both the apex $\left(L_{\mathrm{T}}=22 a\right)$ and the isobar minimum $\left(r_{\mathrm{T}}=2.8 a\right)$ corresponding to a sheath coverage $r_{\mathrm{S}}$. At this magnetic threshold the solution $L_{\mathrm{T}}$ of large apex can be no more followed $\left(r^{\prime 2}\right.$ becomes negative somewhere in the profile !), the apex value jumps down to sheath coverage solution $r_{\mathrm{T}}$; the drop spreads along the wire. Figure 2 shows the spreading of the drop in the vicinity of $H_{\mathrm{T}}$. The magnetic wetting transition is discontinuous but discussion of the possibility of hysteresis effect at the transition would require a more complete calculation of the energy in the vicinity of $H_{\mathrm{T}}$. As $H$ is further increased, the sheath spreads more and more along the wire following the dashed-line of sheath coverage.

2.2.2b Small drop (Figs. 3 and 4). - In this regime the initial drop pressure $P_{\mathrm{i}}$ is much higher than $P_{\mathrm{c}}$; figure 3 displays monotonously decreasing isobars $\left(P>P_{\mathrm{c}}\right)$, and as the drop pressure decreases with $H$, some $P<P_{\mathrm{c}}$ isobars are also included. For monotonous high pressure isobars, there is only one

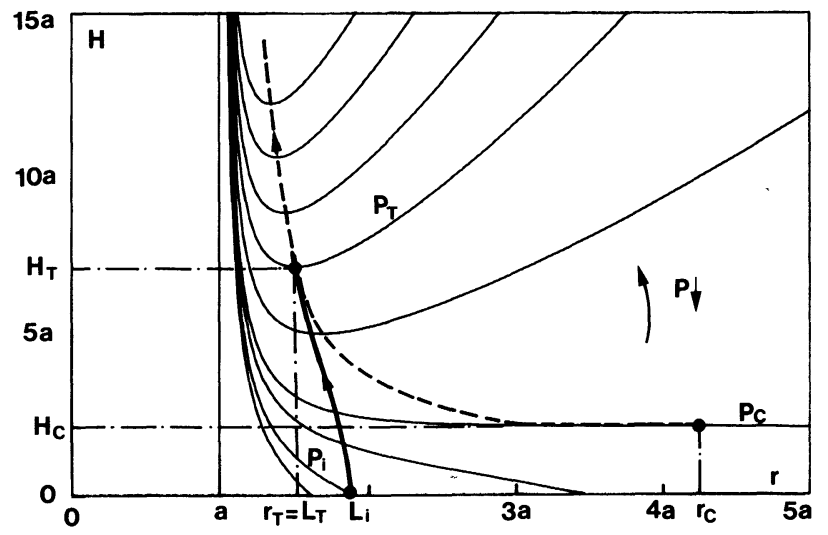

Fig. 3. - Spreading of a small magnetic drop along a conducting wire as magnetic length $H$ is increased (cf. Fig. 4). Thick full-line, full-lines, dashed curve and various subscripts have same meaning as in figure 1 . Numerical values are given in the text and in captions of figure 4. At the threshold, there is no jump in the drop profile $\left(L_{\mathrm{T}}=r_{\mathrm{T}}\right)$ : the magnetic wetting transition is continuous. intersection with horizontal line $H$ : the trivial apex solution $r=L$. The full-line of figure 3 displays the behaviour of a small drop $\left(L_{\mathrm{i}}=1.9 a\right.$ and $P_{\mathrm{i}}=$ $\left.11 P_{\mathrm{c}}\right)$ as $H$ is increased from 0 . The computation of the drop profile (Fig. 4) allows us to follow $L$ and $P$ through volume conservation: the larger $H$ the smaller the apex, the smaller $P$. Indeed, the different decreasing isobars are swept very rapidly, even if these isobars are no more monotonous $\left(P<P_{\mathrm{c}}\right)$ : the apex value $L$ is smaller than the sheath coverage $r_{\mathrm{S}}(H)$ (Eq. (8) is not fullfilled). As $H$ is increased, the flattening of the drop along the wire occurs until a critical magnetic length is reached $\left(H_{\mathrm{T}}=7.1 \mathrm{a}\right.$ in Fig. 3). Then the drop achieves a sheath shape of radius $r_{\mathrm{T}}=r_{\mathrm{S}}$ in its middle. At this magnetic threshold there is no jump in the apex value $\left(L_{\mathrm{T}}=r_{\mathrm{T}}=1.5 a\right.$ and $\left.P_{\mathrm{T}}=-21 P_{\mathrm{c}}\right)$. For small drops the magnetic wetting transition is then continuous. As $H$ is further increased the sheath spreads more and more along the wire following the dashedline of sheath coverage. Corresponding profiles are given in figure 4.

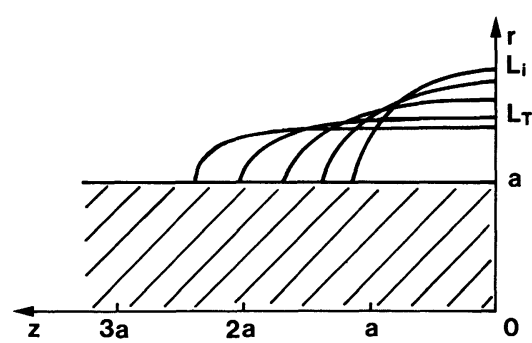

Fig. 4. - Same computed profiles as in figure 2 with a small drop of $L_{\mathrm{i}}=1.9 a$ and $P_{\mathrm{i}}=11 P_{\mathrm{c}}$. The different curves correspond to: $H=0, H=1.8 a, H=4.1 a$, $H=H_{\mathrm{T}}, \quad H=8.8 a \quad\left(H_{\mathrm{T}}=7.1 a, \quad r_{\mathrm{T}}=L_{\mathrm{T}}=1.5 a\right.$, $\left.P_{\mathrm{T}}=-21 P_{\mathrm{c}}\right)$. The contact angle is $\theta=80^{\circ}$.

2.2.2c Critical drop. - Between the two previous regimes $a$ and $b$, there is an intermediate drop size for which the threshold occurs at $P_{\mathrm{T}}=P_{\mathrm{c}}, H_{\mathrm{T}}=$ $H_{\mathrm{c}}, L_{\mathrm{T}}=r_{\mathrm{c}}$ and $r_{\mathrm{T}}=r_{\mathrm{c}}$. It corresponds to the end point of the sheath coverage curve. For such a drop the magnetic wetting transition is turning from continuous to discontinuous.

2.2.2d Infinitely large drop. - Regime a is limited on one side by the critical drop $\left(P_{\mathrm{T}}=P_{\mathrm{c}}\right)$ and on the other one by the infinitely large drop $(L=\infty$ and $P=0$ whatever $H)$. The magnetic wetting transition is there indeed discontinuous and corresponds to section 2.1 calculations of the ferrofluid ascension along a wire with $p_{0}=0$.

\section{Experimental observation.}

A first experimental evidence of this drop-sheath transition is presented in picture 1 , it corresponds only to preliminary experiments. The magnetic 


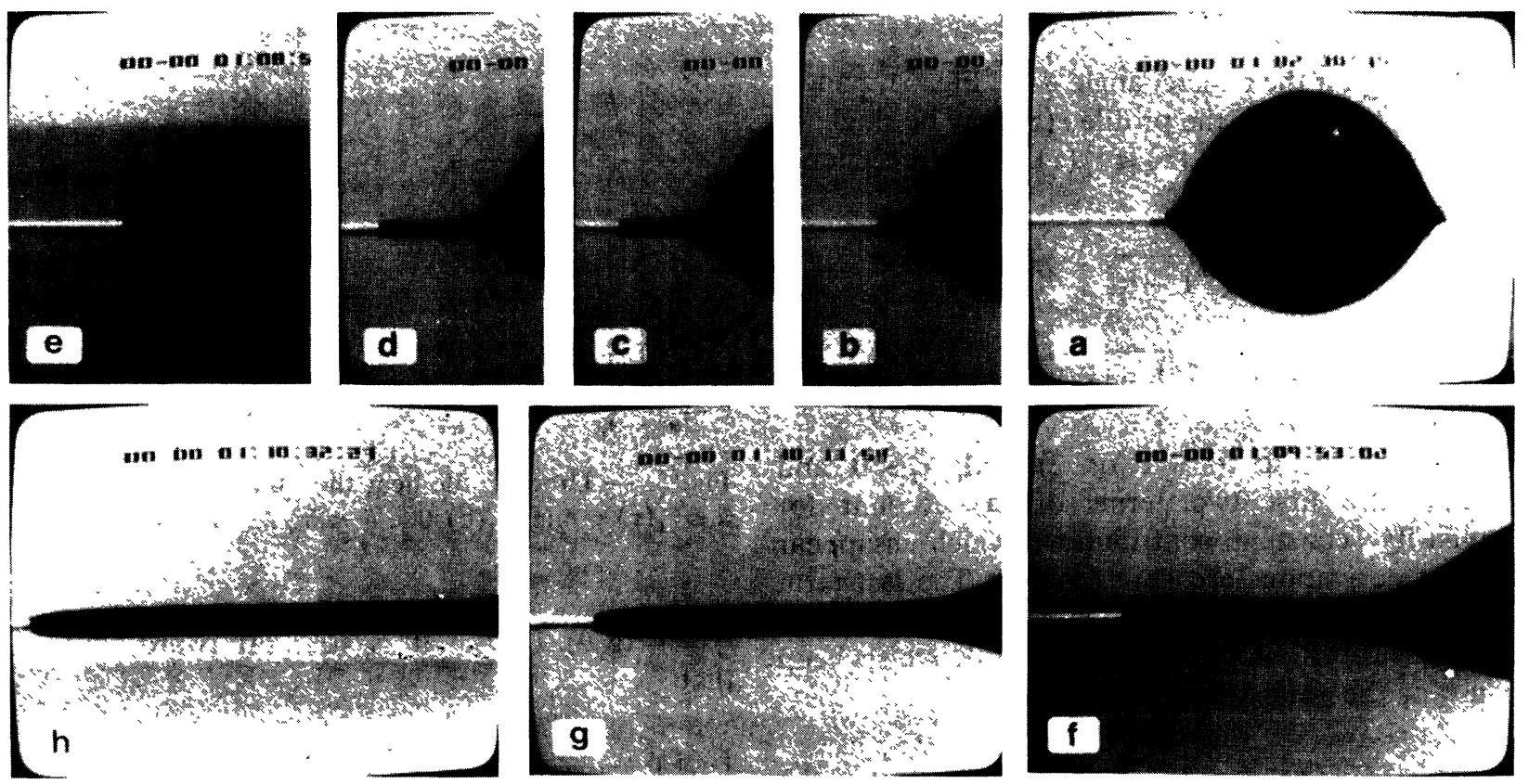

Picture 1. - Pictures of the spreading of a ferrofluid along a conducting wire of radius $a=50 \mu \mathrm{m}$. The current $I$ in the wire determines the magnetic length: (a) $I=0.4 \mathrm{~A}, H=0.3 a ;$ (b) $I=0.7 \mathrm{~A}, H=0.9 a ;$ (c) $I=0.75 \mathrm{~A}$, $H=a$; (d) $I=0.8 \mathrm{~A}, H=1.2 a$; (e) $I=1.1 \mathrm{~A}, H=2.2 a$; (f), (g), (h) $I=1.2 \mathrm{~A}, H=2.6 a$. Asymmetry in the drop profile is due to an imperfect density adjustement between the ferrofluid and the surrounding liquid.

liquid is an ionic ferrofluid $[12,13]$ : a colloidal dispersion of $\mathrm{FeCoO}_{4}$ macroanions of typical size $10 \mathrm{~nm}$ in an alkalyne aqueous medium. Such ionic ferrofluids exhibit a large magnetic relative permeability in low fields, for the present sample $\mu_{\mathrm{r}}=1+\chi=5.6$. The surrounding fluid is freon and the two fluids have been chosen in order to match their densities $\left(\Delta \rho<10 \mathrm{~kg} \cdot \mathrm{m}^{-3}\right)$. Their interfacial tension is $\gamma=0.8 \mathrm{~mJ} . \mathrm{m}^{-2}$. We use a copper wire of radius $a=50 \mu \mathrm{m}$. For $I=1 A$ the magnetic field at the wire surface $\left(B=\mu_{0} I / 2 \pi a\right)$ is $4 \times$ $10^{-3} \mathrm{~T}$ and corresponds to a magnetic length $\left(H=\mu_{0} \chi I^{2} / 8 \pi^{2} \gamma\right) H=90 \mu \mathrm{m}=1.8 a$.

The drop of picture 1 is comparable to the large drop of figures 1 and 2 : its initial apex for $I=0$ is $L_{\mathrm{i}}=26 a$. Various pictures correspond to various current intensities $I$ in the wire and thus to various $H$ values. Pictures $1 \mathrm{a}$ to $1 \mathrm{e}$ are equilibrium shapes (Fig. $1 \mathrm{a}: I=0.4 \mathrm{~A}, H=0.3 a$; Fig. $1 \mathrm{~b}$ : $I=0.7 \mathrm{~A}, H=0.9 a$; Fig. $1 \mathrm{c}: I=0.75 \mathrm{~A}, H=a$; Fig. $1 \mathrm{~d}: I=0.8 \mathrm{~A}, H=1.2 a$; Fig. $1 \mathrm{e}: I=1.1 \mathrm{~A}$, $H=2.2 a$ ). In these pictures, the shape of the drop is similar to those of figure 2 , below the magnetic threshold. There is an experimental difficulty, already pointed out in the previous experiment of ascension along a vertical wire [8]: the apparent contact angle $\theta$ varies from picture 1 a to picture $1 \mathrm{~d}$, it may be due to hysteresis effects induced by the wire surface roughness. Anyway above $I=0.8 \mathrm{~A}$ $(H>1.2 a$, Fig. $1 \mathrm{~d}$ to Fig. $1 \mathrm{~h}$ ), $\theta$ remains constant and equal to $80^{\circ}$. For this contact angle, the magnetic wetting transition is predicted (cf. Fig. 1 and Fig. 2) to occur for $H_{\mathrm{T}}=2.34 a$, to be discontinuous and to lead to a sheath coverage of thickness $r_{\mathrm{T}}=2.8 a$. These three points are closely compatible with our experimental observations. Pictures $1 \mathrm{f}$ to $1 \mathrm{~h}$ are a sequence of dynamical shapes for $H=2.6 a$ (i.e. $I=1.2 \mathrm{~A}$ ) that is just above the predicted threshold.

If such a spectacular profile jump is observed with large drops $\left(L_{\mathrm{i}} \simeq 26 a\right)$, on the contrary small drops $\left(L_{\mathrm{i}} \simeq 2 a\right)$ experimentally experience a continuous deformation from a drop-shape to a sheath-shape, in agreement with predictions of figures 3 and 4.

\section{Conclusion.}

We have discussed the spreading of a non wetting liquid magnetic drop along a wire. The control parameter of the ferrofluid spreading is the magnetic length which is related to the current in the conducting wire. For a threshold magnetic length we predict a spreading of the drop into a sheath coverage. This magnetic wetting transition is analogous to the wetting transition along a fiber predicted for totally wetting fluid in presence of Van der Waals forces. We also discuss the continuous or discontinuous character of the transition depending on the drop size.

\section{Acknowledgments.}

We are greatly indebted to $F$. Tourinho and R. Massart for providing us with ionic ferrofluid samples and to A. Levelut for helpful discussions. 


\section{Appendix I.}

Depending on the contact angle $\theta$ (cf. Fig. 5), $r$ and $z$ being both positive, the profile $r(z)$ may be single or double valued : $a \leqslant r_{1}(z) \leqslant r_{2}(z)$. The free energy of the system is :

$$
\begin{aligned}
F=2 \pi \int \mathrm{d} z & \left(\gamma r_{1} \sqrt{1+r_{1}^{\prime 2}}+\right. \\
& \left.+\gamma r_{2} \sqrt{1+r_{2}^{\prime 2}}-\gamma H \log \frac{r_{2}}{r_{1}}\right)
\end{aligned}
$$

where $r_{i}=\mathrm{d} r_{i} / \mathrm{d} z \quad(i=1$ or 2$)$ with $r_{1}^{\prime}>0$ and $r_{2}^{\prime}<0$. The first two terms of (a) account for capillarity, constant contributions which disappear in next derivations are here omitted. The last term accounts for magnetism. Free energy $F$ must be minimal for all variations of the profile which keep constant the total volume of the drop :

$$
\Omega=\pi \int\left(r_{2}^{2}-r_{1}^{2}\right) \mathrm{d} z
$$

Using a Lagrange multiplier $p_{0}$, which may be interpreted as the pressure difference between the magnetic fluid and the surrounding one, we minimize $\mathcal{L}=F-p_{0} \Omega$ at constant volume $\Omega$. As $z$ does not explicitely appear in $\mathcal{L}$, the equilibrium profile is obtained through relations $\mathcal{L}-r_{i}^{\prime}\left(\partial \mathcal{L} / \partial r_{i}^{\prime}\right)=$ cst. $(i=1$ or 2$)$. One obtains :

$$
(-1)^{i} \frac{r_{i}}{\sqrt{1+r_{i}^{\prime 2}}}-\dot{H} \log r_{i}-\frac{p_{0} r_{i}^{2}}{2 \gamma}=\text { Cst . }
$$

The boundary condition at the wire surface is
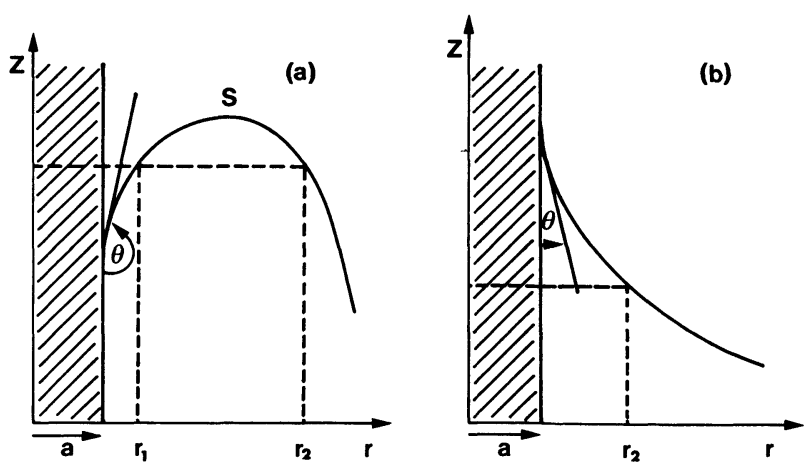

Fig. 5. - Drop profile near the tip : (a) $\pi / 2 \leqslant \theta<\pi$ : $a \leqslant r_{1}(z) \leqslant r_{2}(z)$; (b) $0<\theta \leqslant \pi / 2: r_{1}(z) \equiv a$.

$r_{i}^{\prime}=-\operatorname{tg} \theta, r_{i}=a$. It is straightforward to verify that :

$$
\begin{aligned}
&(-1)^{i} \frac{r_{i}}{\sqrt{1+r_{i}^{\prime 2}}}-a \cos \theta-H \log \frac{r_{i}}{a}- \\
&-\frac{p_{0}}{2 \gamma}\left(r_{i}^{2}-a^{2}\right)=0
\end{aligned}
$$

- with $i=2$ for $0<\theta \leqslant \pi / 2$ : as $r^{\prime}(a)<0$, $r_{1}$ is equal to a all along the profile and $r(z) \equiv$ $r_{2}(z)$ on the whole range;

- with $i=1$ or 2 for $\pi / 2 \leqslant \theta<\pi$ : the magnetic profile $r(z)$ is first equal to $r_{1}(z)$ near the wire $\left(r^{\prime}(a)>0\right)$ and then equal to $r_{2}(z)$ after the point $S$ where $r_{1}=r_{2}$ and $r_{1}^{\prime}=r_{2}^{\prime}=\infty$ (cf. Fig. 5).

Expression (d) is the general formulation of expression (4) ; other expressions from (5) to (11) are valid whatever the $\theta$ value $(0<\theta<\pi)$.

\section{References}

[1] Young, T., Philos. Trans. R. Soc. London 95 (1805) 65.

[2] De Gennes, P. G., Rev. Mod. Phys. 57 (1985) 827 and references therein.

[3] JoAnNy, J. F., Thèse de Doctorat d'Etat, Université P. et M. Curie, Paris (1985);

JoAnny, J. F., DE Gennes, P. G., C. R. Acad. Sci. Paris 299 II (1984) 279 ; 299 II (1984) 605.

[4] Huh, C., SCRIVEn, L. E., J. Colloid Interface Sci. 35 (1971) 85.

[5] Brochard, F., J. Chem. Phys. 84 (1986) 4664.

[6] Di Meglio, J. M., C. R. Acad. Sci. Paris 303 II (1986) 437.
[7] Di Meglio, J. M., Brochard, F., Quere, D., Revue Phys. Appl. 23 (1988).

[8] BACRI, J. C., PERzYNSKI, R., SAlin, D., TOURINHo, F., Europhys. Lett. 5 (1988) 547.

[9] Pincus, P., Biophysical effects of steady magnetic fields (Springer Verlag) 1986.

[10] ARKhipenKI, V. I., BARKOV, Yu. D., BASHTOVoI, V. G., Magn. Gidrodin. 3 (1980) 3.

[11] BERKOVSKY, B., BASHTOVOI, V., IEEE Trans. Magn. MAG-16 (1980) 288.

[12] Rosensweig, R. E., Ferrohydrodynamics (Cambridge University Press) 1985.

[13] Massart, R., IEEE Trans. Magn. MAG-17 (1981) 1247. 